\title{
Energy-efficient estimation of a MIMO channel
}

\author{
Camila Muñoz and Christian Oberli
}

\begin{abstract}
Exploiting the benefits of multiple antenna technologies is strongly conditioned on knowledge of the wireless channel that affects the transmissions. To this end, various channel estimation algorithms have been proposed in the literature for multiple-input multiple-output (MIMO) channels. These algorithms are typically studied from a perspective that does not consider constraints on the energy consumption of their implementation. This article proposes a methodology for evaluating the total energy consumption required for transmitting, receiving, and processing a preamble signal in order to produce a channel estimate in multiple antenna systems. The methodology is used for finding the training signals that minimize the energy consumption for attaining given mean square estimation error. We show that the energy required for processing the preamble signal by executing the estimation algorithms dominates the total energy consumed by the channel estimation process. Therefore, algorithm simplicity is a key factor for achieving energy-efficient channel acquisition. We use our method for analyzing the widely used least squares and minimum mean square error (MSE) estimation algorithms and find that both have a similar energy consumption when the same MSE estimation is targeted.
\end{abstract}

Keywords: MIMO channel estimation, Energy efficiency

\section{Introduction}

Multiple-input multiple-output (MIMO) communication techniques have been incorporated into different wireless systems due to their capability for allowing higher data rates (multiplexing gain) or for increasing link reliability (diversity gain). However, recent studies have shown that MIMO techniques can be used alternatively for reducing energy consumption in comparison to a single-input single-output (SISO) link that attains the same data rate and link reliability. In [1], when the link distance is larger than a given threshold, data transmission using a $2 \times 2$ MIMO system with Alamouti space-time coding was shown to be more energy-efficient than an equivalent SISO system. A detailed energy consumption model for an $N \times N$ singular value decomposition-based MIMO system is proposed in [2]. The model includes retransmission statistics and shows that for a given link distance and number of channels used exist a single optimal radiation power level at which the mean energy consumption required to transmit a bit correctly is minimized.

But the use of MIMO is strongly conditioned on knowing the wireless channel, which the above contributions

\footnotetext{
${ }^{*}$ Correspondence: cmunoz1@uc.cl

Department of Electrical Engineering, Pontificia Universidad Católica de Chile, Santiago, Chile
}

assume perfectly known. This knowledge is typically obtained by transmitting a known training preamble that allows the receiver to estimate the channel by executing an estimation algorithm.

The design of training preambles for channel estimation has not yet been studied well in terms of energy efficiency. Typically, the design of the preamble signals focuses on minimizing the channel estimation error [3] or on maximizing the channel capacity under imperfect channel knowledge [4]. Furthermore, existing models of MIMO energy consumption as the ones in [1,2] ignore the energy required for transmitting, receiving, and processing a preamble signal. In fact, MIMO channel estimation can be a significant part of the baseband processing energy consumption because the algorithms usually perform complex algebraic operations.

In this article, we present a method for comparing the energy efficiency of different channel estimation algorithms. We formulate an energy consumption model that allows to find the training signals that minimize the energy consumption of the algorithms given a mean square error (MSE) of estimation. Particularly, we study the minimum MSE (MMSE) and least squares (LS) channel estimation algorithms and optimize their respective preambles for minimum energy consumption at a given target MSE. We

\section{黛 Springer}

(c) 2012 Muñoz and Oberli; licensee Springer. This is an Open Access article distributed under the terms of the Creative Commons Attribution License (http://creativecommons.org/licenses/by/2.0), which permits unrestricted use, distribution, and reproduction in any medium, provided the original work is properly cited. 
show that their optimal energy consumption difference is negligible.

The rest of the article is organized as follows: Section 2 describes the energy consumption model for channel estimation algorithms. Section 3 examines the LS and MMSE algorithms and details their energy consumption and MSE. Section 4 formulates and solves the optimization problem that allows to find the optimal training signals. Section 5 provides numerical results and Section 6 summarizes our conclusions.

Notation: $\mathbf{x}^{H}$ denotes the conjugate transpose operation over $\mathbf{x},\|\mathbf{x}\|$ is the norm of vector $\mathbf{x}, \mathbb{E}\{\cdot\}$ indicates expected value and $\mathbf{I}_{M}$ is the $M \times M$ identity matrix. The superscript check ${ }^{2}$ denotes that the variable corresponds to a single branch of either the transmitter or receiver.

\section{Energy consumption model}

Our goal is to minimize the energy consumption required for producing a channel estimate with a given estimation quality. For this purpose, we formulate a model that includes the energy consumption of all the components involved in the channel estimation process.

We consider a generic low-power MIMO transceiver architecture for the preamble communication as shown in Figure 1, with $N_{t}$ transmit and $N_{r}$ receive antennas. It is to be noted that data processing blocks such as channel encoder, modulator, channel decoder, and demodulator are not included because they do not participate in the channel estimation process.

In the sequel, we detail each source of the energy consumption.

1. Start-up energy consumption, $\mathcal{E}_{\mathrm{ST}}$ We assume that the transmitter and receiver are by default in a low-power consumption (sleep) mode. Hence, they must be brought online before they can communicate the preamble. We denote as $\mathcal{E}_{\mathrm{ST}}$ the energy required to start-up the transceivers, which is dominated by the stabilization of the frequency synthesizer [5]. If this component consumes a power $P_{\text {syn }}$ has a settling time $T_{\text {tr }}$ and is shared among all branches (either transmitting or receiving), then the start-up energy of two frequency synthesizers can be expressed as

$$
\mathcal{E}_{\mathrm{ST}}=2 P_{\text {syn }} T_{\text {tr }} .
$$

2. Energy consumption of the transmitter electronics, $\mathcal{E}_{\text {el,tx }}$ It represents the energy consumption of the digital-to-analog converters (DAC), filters, and mixers of the transmitter. These components consume energy for each transmitted preamble
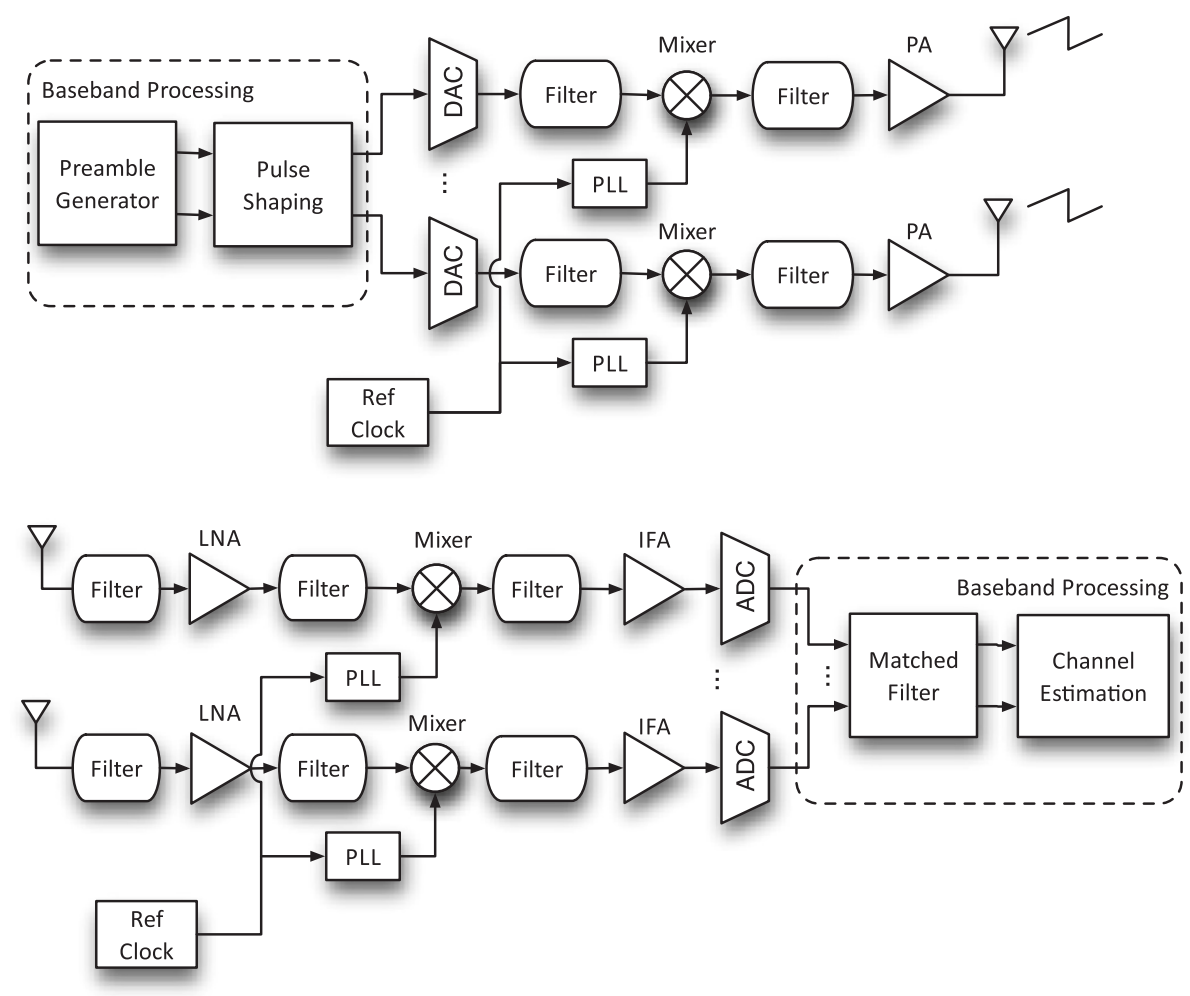

Figure 1 Block diagram of a generic MIMO transceiver used during the preamble communication (top, transmitter; bottom, receiver). 
symbol. We define a binary variable $s_{(n, t)}$ that indicates if transmitter branch $n$ transmits a preamble symbol during symbol time $t$, with $n=1, \ldots, N_{t}$ and $t=1, \ldots, N_{p}$. Thus, the total energy consumed by these components is given by

$$
\mathcal{E}_{\mathrm{el}, \mathrm{tx}}=T_{s}\left(\check{P}_{\mathrm{el}, \mathrm{tx}} \sum_{n=1}^{N_{t}} \sum_{t=1}^{N_{p}} s_{(n, t)}+P_{\mathrm{syn}} N_{p}\right),
$$

where $T_{S}$ is the symbol period and $\check{P}_{\text {el,tx }}=\check{P}_{\text {DAC }}+\check{P}_{\text {filter }}+\check{P}_{\text {mixer }}$ represents the power consumption of DAC, filters and mixer of each transmitter branch. This model allows for each antenna to transmit a different and arbitrary sequences of training symbols, which can include silences.

3. Energy consumption due to electromagnetic radiation, $\mathcal{E}_{\mathrm{PA}}$

Each preamble symbol is broadcast from a transmitting antenna with a transmission power $\check{P}_{\mathrm{tx}}$ provided by the respective power amplifier (PA). The PA's power consumption is modeled by

$$
\check{P}_{\text {PA }}=\frac{\xi}{\eta} \check{P}_{\mathrm{tx}}
$$

where $\xi$ is the peak-to-average ratio of the transmitted signal and $\eta$ is the drain efficiency of the PA [5]. The energy consumed jointly by all PA's is

$$
\mathcal{E}_{\mathrm{PA}}=\frac{\xi}{\eta} T_{s} \sum_{n=1}^{N_{t}} \sum_{t=1}^{N_{p}} \check{P}_{\mathrm{tx}(n, t)} S_{(n, t)},
$$

where $\check{P}_{\mathrm{tx}(n, t)}$ is the irradiated power of the training symbol transmitted by the antenna $n$ during the symbol time $t$, with $n=1, \ldots, N_{t}$ and $t=1, \ldots, N_{p}$. This model allows for arbitrary transmission power per preamble symbol.

4. Energy consumption of the receiver electronics, $\mathcal{E}_{\mathrm{el}, \mathrm{rx}}$ It represents the energy consumption of the components that remain energized during the reception time of the preamble, which is equal to $N_{p} T_{s}$. Thus,

$$
\mathcal{E}_{\mathrm{el}, \mathrm{rx}}=N_{p} T_{s}\left(\check{P}_{\mathrm{el}, \mathrm{rx}} N_{r}+P_{\mathrm{syn}}\right)
$$

where $\check{P}_{\text {el,rx }}=\check{P}_{\text {ADC }}+\check{P}_{\text {filter }}+\check{P}_{\text {mixer }}+\check{P}_{\text {LNA }}+\check{P}_{\text {IFA }}$ represents the power consumption of analog-to-digital converter (ADC), filters, mixer, low-noise amplifier (LNA) and intermediate frequency amplifier (IFA) of each receiving branch. We will assume that filters and mixers at the receiver consume the same power as these components at the transmitter.
5. Energy consumption due to channel estimation, $\mathcal{E}_{\text {estim }}$

Every time a packet is received, the channel estimation engine performs $K$ different kinds of arithmetic operations, each of which has an energy consumption $\mathcal{E}_{k}$, with $k=1, \ldots, K$ and is performed $n_{k}$ times during the execution of the entire channel estimation algorithm. Thus,

$$
\mathcal{E}_{\text {estim }}=\sum_{k=1}^{K} n_{k} \mathcal{E}_{k} .
$$

If the operations are performed by an arithmetic processing unit (APU), the energy consumption of the $k$ th operation can be modeled as [6]

$$
\mathcal{E}_{k}=V_{\mathrm{dd}} I_{o} \Delta t_{k},
$$

where $V_{\mathrm{dd}}$ is the APU operating voltage, $I_{o}$ is the average current during the execution time of the arithmetic operations. It is to be noted that $I_{o}$ depends on $V_{\mathrm{dd}}$ and on the clock frequency, $f_{\mathrm{APU}}$. $\Delta t_{k}$ is the time required for executing the $k$ th operation. It is related to the number of clock cycles, $c_{k}$, required by the operation and to $f_{\mathrm{APU}}$ as follows:

$$
\Delta t_{k}=\frac{c_{k}}{f_{\mathrm{APU}}} .
$$

Replacing these terms in (6), the energy required for estimating the channel is given by

$$
\mathcal{E}_{\mathrm{estim}}=\frac{V_{\mathrm{dd}} I_{o}}{f_{\mathrm{APU}}} \sum_{k=1}^{K} n_{k} c_{k}
$$

The sum of the energies (1), (2), (4), (5), and (9) gives the total energy consumed by the communication process in order to produce a channel estimate. It is given by

$$
\begin{aligned}
\mathcal{E}_{\text {total }}= & 2 P_{\text {syn }} T_{\text {tr }}+T_{s}\left(\check{P}_{\text {el, } \mathrm{tx}} \sum_{t=1}^{N_{t}} \sum_{p=1}^{N_{p}} s_{(t, p)}+P_{\text {syn }} N_{p}\right) \\
& +T_{s} N_{p}\left(\check{P}_{\mathrm{el}, \mathrm{rx}} N_{r}+P_{\mathrm{syn}}\right)+T_{s} \frac{\xi}{\eta} \sum_{t=1}^{N_{t}} \sum_{p=1}^{N_{p}} \check{P}_{\mathrm{tx}(t, p)} s_{(t, p)} \\
& +\frac{V_{\mathrm{dd}} I_{o}}{f_{\mathrm{APU}}} \sum_{k=1}^{K} n_{k} c_{k} .
\end{aligned}
$$

We now turn our attention to the estimation problem, focusing on the number of arithmetic operations required by various common channel estimation algorithms.

\section{Channel estimation algorithms}

In this section, we characterize the LS and MMSE channel estimation algorithms by their complexity of implementation and associated MSE performance. This requires to 
formulate a signal model that describes the communication of the preamble and to determine the arithmetic operations that each algorithm performs.

It is to be noted that the problem of channel estimation in a MIMO system with $N_{t}$ transmit and $N_{r}$ receive antennas is, in practice, a set of $N_{t}$ independent single-input multiple-output problems, one per transmitter branch. This follows from observing that it cannot be assumed $a$ priori that the $N_{r} \times N_{t}$ channel coefficients that compose the MIMO channel are correlated in any particular fashion. In the most unfavorable case, observations from one transmitter branch contain no information about channel parameters of another one, and transmitting information simultaneously from more than one branch provides no benefit and increases complexity. This implies that an $N_{r} \times N_{t}$ channel matrix $\mathbf{H}$ can be estimated sequentially by columns using a preamble in which only one antenna simultaneously transmits a training sequence, as shown in [7] (Figure 2). In this case, the condition $\sum_{t=1}^{N_{p}} s_{(n, t)}=$ $N_{p} / N_{t}$ must hold for each transmitter branch so that column of $\mathbf{H}$ is estimated using the same number of pilot symbols.

The estimation of the $j$ th column of $\mathbf{H}$ only requires the information of the received signal that was generated during the time when the $j$ th branch transmitted its preamble. The received signal can be expressed as

$$
\mathbf{Y}_{j}=\frac{1}{\sqrt{A d^{\alpha}}} \mathbf{h}^{j} \mathbf{p}_{j}+\mathbf{V}_{j}
$$

where $A d^{\alpha}$ represents the path loss, with $d$ the link distance, $\alpha$ the path loss exponent, and $A$ a parameter that depends on the transmitter and receiver antenna gains and the transmission wavelength ( $A$ may include shadow fading) [8]. $\mathbf{p}_{j}$ is the preamble sequence transmitted by the $j$ th branch. $\mathbf{h}^{j}$ is the $j$ th column of $\mathbf{H}$ and its elements represent the small scale fading of the MIMO channel. We assume that the wireless channel is static and flat fading. $\mathbf{V}_{j}$ is a matrix of independent complex Gaussian random variables with zero mean and variance $\sigma_{n}^{2}$, representing additive white Gaussian noise. The variance $\sigma_{n}^{2}$ depends on the transmission bandwidth $W$, on the receiver noise figure, $N_{f}$, and on the link margin $M_{l}[1]$.

The receiver compensates the path loss using an LNA. Thus, the signal observed by the channel estimator is

$$
\mathbf{S}_{j}=\mathbf{h}^{j} \mathbf{p}_{j}+\sqrt{A d^{\alpha}} \mathbf{V}_{j}
$$

In the following, we analyze the energy consumption and MSE of the LS and MMSE algorithms.

\subsection{LS algorithm}

Given $\mathbf{S}_{j}$ and knowledge of $\mathbf{p}_{j}$, the LS estimator for the $j$ th column of $\mathbf{H}$ is [9]

$$
\hat{\mathbf{h}}_{\mathrm{LS}}^{j}=\mathbf{S}_{j} \frac{\mathbf{p}_{j}^{H}}{\left\|\mathbf{p}_{j}\right\|^{2}} .
$$

As $\mathbf{p}_{j}^{H} /\left\|\mathbf{p}_{j}\right\|^{2}$ is known a priori, the estimation only requires the product between the $N_{r} \times \frac{N_{p}}{N_{t}}$ matrix $\mathbf{S}_{j}$ and an $\frac{N_{p}}{N_{t}} \times 1$ vector. This takes $\frac{N_{r} N_{p}}{N_{t}}$ complex products and $N_{r}\left(\frac{N_{p}}{N_{t}}-1\right)$ complex sums each time a column of $H$ is estimated. Standard implementations of these complex operations require four real products and two real sums for each complex product, and two real sums for each complex sum [10]. Then, performing the estimation (13) for $N_{t}$ columns of $\mathbf{H}$ requires $2 N_{r}\left(2 N_{p}-N_{t}\right)$ real additions and $4 N_{r} N_{p}$ real multiplications.

Finally, assuming that every pilot symbol is transmitted with power $P_{\mathrm{tx}}$, the energy consumption model (10) for the case of the LS algorithm becomes

$$
\begin{aligned}
\mathcal{E}_{\mathrm{LS}}= & 2 P_{\mathrm{syn}} T_{\mathrm{tr}}+\left(\check{P}_{\mathrm{el}, \mathrm{tx}}+N_{r} \check{\mathrm{P}}_{\mathrm{el}, \mathrm{rx}}+2 P_{\mathrm{syn}}\right) T_{s} N_{p} \\
& +\frac{\xi}{\eta} T_{s} N_{p} P_{\mathrm{tx}}+\frac{2 V_{\mathrm{dd}} I_{o} N_{r}}{f_{\mathrm{APU}}}\left[\left(2 N_{p}-N_{t}\right) c_{\mathrm{sum}}+2 N_{p} c_{\mathrm{prod}}\right] .
\end{aligned}
$$

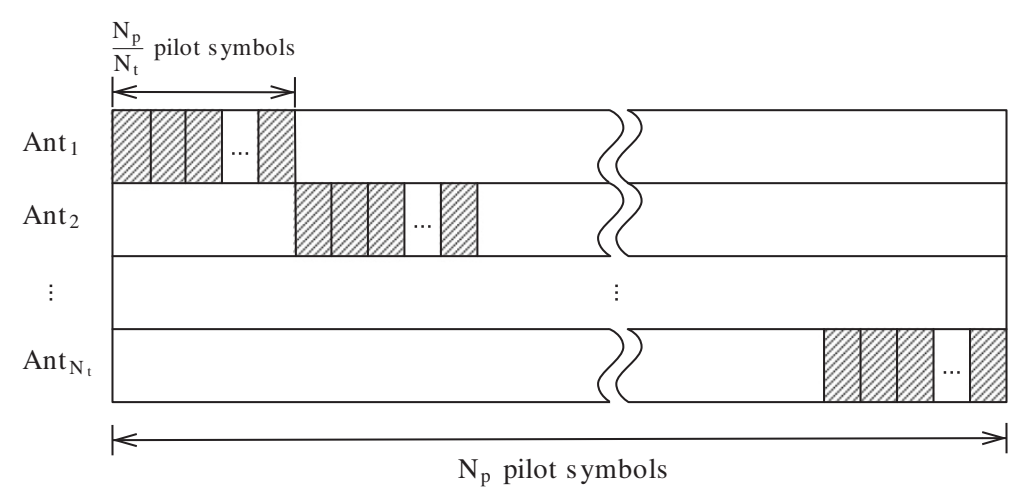

Figure 2 Distribution of pilot symbols per antenna, with a time-orthogonal preamble. 
The MSE of the LS algorithm is [9]

$$
\operatorname{MSE}_{\mathrm{LS}}=\frac{A d^{\alpha} \sigma_{n}^{2} N_{r} N_{t}^{2}}{N_{p} P_{\mathrm{tx}}},
$$

which uses the fact that each column of $\mathbf{H}$ is estimated with $\frac{N_{p}}{N_{t}}$ equal power pilot symbols.

\subsection{MMSE algorithm}

The MMSE estimator for the $j$ th column of $\mathbf{H}$ is [9]

$$
\hat{\mathbf{h}}_{\mathrm{MMSE}}^{j}=\mathbf{S}_{j} \mathbf{p}_{j}^{H} \frac{\mathbf{R}_{H}^{(j)}}{N_{r} A d^{\alpha} \cdot \sigma_{n}^{2}+\mathbf{R}_{H}^{(j)} \cdot\left\|\mathbf{p}_{j}\right\|^{2}},
$$

where $\mathbf{R}_{H}^{(j)}$ is the $j$ th element of the diagonal of the correlation matrix $\mathbf{R}_{H}=\mathbb{E}\left\{\mathbf{H}^{H} \mathbf{H}\right\}$. We assume that $\mathbf{R}_{H}$ and $\sigma_{n}^{2}$ are perfectly known but can change from one transmission to the next. Therefore, the quotient in (16) has to be calculated with each new estimation of $\hat{\mathbf{h}}_{\mathrm{MMSE}}^{j}$. This requires to perform two products, shown with '.' in (16), the sum in the denominator and the division plus the evaluation of $\mathbf{S}_{j} \mathbf{p}_{j}^{H}$, as in the LS case. The total number of real arithmetic operations required by this algorithm are shown in Table 1.

Replacing the number of arithmetic operations of Table 1 in (10), the energy consumption model for MMSE algorithm is

$$
\begin{aligned}
\mathcal{E}_{\mathrm{MMSE}}= & 2 P_{\mathrm{syn}} T_{\mathrm{tr}}+\left(\check{P}_{\mathrm{el}, \mathrm{tx}}+N_{r} \check{P}_{\mathrm{el}, \mathrm{rx}}+2 P_{\mathrm{syn}}\right) T_{s} N_{p} \\
& +\frac{\xi}{\eta} T_{s} N_{p} P_{\mathrm{tx}}+\frac{V_{\mathrm{dd}} I_{o}}{f_{\mathrm{APU}}}\left[\left(4 N_{r} N_{p}+9 N_{t}\right) c_{\mathrm{sum}}\right. \\
& \left.+\left(4 N_{r} N_{p}+4 N_{r} N_{t}+14 N_{t}\right) c_{\mathrm{prod}}+2 N_{t} c_{\mathrm{div}}\right]
\end{aligned}
$$

where $c_{\text {sum }}, c_{\text {prod }}$, and $c_{\text {div }}$ describe the number of cycles required for performing a sum, product, and division, respectively.

The MSE of the MMSE estimation algorithm is [9]

$$
\operatorname{MSE}_{\mathrm{MMSE}}=A d^{\alpha} \sigma_{n}^{2} N_{r} N_{t} \sum_{j=1}^{N_{t}} \frac{\mathbf{R}_{H}^{(j)}}{A d^{\alpha} \sigma_{n}^{2} N_{r} N_{t}+\mathbf{R}_{H}^{(j)} N_{p} P_{\mathrm{tx}}} .
$$

It is to be noted that (14) and (17) share a common structure, namely

$$
\mathcal{E}=k_{1}+k_{2} N_{p}+k_{3} N_{p} P_{\mathrm{tx}}
$$

Table 1 Number of instructions required by the MMSE estimator

\begin{tabular}{ll}
\hline Operation & Number of instructions \\
\hline Sum & $n_{\text {sum }}=4 N_{r} N_{p}+9 N_{t}$ \\
Product & $n_{\text {prod }}=4 N_{r} N_{p}+4 N_{r} N_{t}+14 N_{t}$ \\
Division & $n_{\text {div }}=2 N_{t}$ \\
\hline
\end{tabular}

with $k_{1}, k_{2}$, and $k_{3}$ given in Table 2 . The constant $k_{1}$ is a base energy consumption independent of the number of pilot symbols transmitted. This constant is larger in the case of MMSE algorithm. The term $k_{2} N_{p}$ represents the energy consumption due to electronics and baseband processing and depends linearly on the number on pilot symbols transmitted. $k_{3} N_{p} P_{\mathrm{tx}}$ represents the energy consumption due to electromagnetic radiation. The energy consumption increases as a function of $N_{p}$ and $P_{\mathrm{tx}}$ has the same slope for MMSE and LS estimation because $k_{2}$ and $k_{3}$ are equal in both cases.

\section{Minimization of the channel estimation energy consumption}

In this section, we formulate and solve the optimization problem of minimizing the total energy consumption required for carrying out the LS and MMSE channel estimation algorithms as a function of the number of pilot symbols $N_{p}$ and of the transmission power $P_{\mathrm{tx}}$. Expression (19) is the objective function of the minimization problem.

We assume the following

A1: The transceivers have $N_{r}=N_{t}=N$ antennas and $P_{\text {syn }}, T_{\text {tr }}, \breve{P}_{\text {el,tx }}, \breve{P}_{\text {el, rx }}, V_{\mathrm{dd}}, I_{o}, f_{\mathrm{APU}}, c_{\mathrm{sum}}, c_{\mathrm{prod}}, c_{\mathrm{div}}, \eta$, and $W$ are known parameters.

A2: The path loss parameter $A$, link distance $d$, and path loss exponent $\alpha$ are given and the matrix $H$ is an uncorrelated flat fading MIMO Rayleigh channel with $\mathbf{R}_{H}=\mathbb{E}\left\{\mathbf{H}^{H} \mathbf{H}\right\}=N \mathbf{I}_{N}$.

In addition, we consider the following restrictions:

R1: In order to ensure a given estimation error, expressions (15) and (18) are upper-bounded by $\epsilon_{\max }$.

R2: The number of pilot symbols $N_{p}$ should be equal or greater than $N$, so that at least one pilot symbol is transmitted by each antenna.

R3: Transmission power is constant $P_{\mathrm{tx}}$ (thus, $\xi=1$ ) and limited to $P_{\max }$.

Considering these assumptions and restrictions along with the expressions (15), (18), and (19), our optimization problem can be written in general as

$$
\begin{array}{cl}
\underset{N_{p}, P_{\mathrm{tx}}}{\operatorname{minimize}} & k_{1}+k_{2} N_{p}+k_{3} N_{p} P_{\mathrm{tx}} \\
\text { subject to } & -N_{p} P_{\mathrm{tx}}+k_{4} \leq 0 \\
& -N_{p}+N \leq 0 \\
& P_{\mathrm{tx}}-P_{\max } \leq 0,
\end{array}
$$


Table 2 Constants of the energy model (19)

\begin{tabular}{|c|c|c|}
\hline & LS & MMSE \\
\hline$k_{1}$ & $2 P_{\text {syn }} T_{\mathrm{tr}}-2 \frac{V_{\text {dd }} l_{0}}{f_{\mathrm{APU}}} N_{r} N_{t} C_{\text {sum }}$ & $2 P_{\text {syn }} T_{\text {tr }}+\frac{V_{\text {dd }} l_{o}}{f_{\mathrm{APU} U}} N_{t}\left[9 c_{\text {sum }}+\left(4 N_{r}+14\right) c_{\text {prod }}+2 c_{\text {div }}\right]$ \\
\hline$k_{2}$ & $T_{s}\left(\check{P}_{\mathrm{el}, t \mathrm{tx}}+N_{r} \check{P}_{\mathrm{el}, 1 \mathrm{rx}}+2 P_{\text {syn }}\right)+\frac{4 V_{\mathrm{dd}} l_{0} N_{r}}{f_{\text {APu }}}\left(c_{\text {sum }}+c_{\text {prod }}\right)$ & $T_{s}\left(\check{P}_{\mathrm{el}, \mathrm{tx}}+N_{r} \breve{P}_{\mathrm{e} l, r x}+2 P_{\text {syn }}\right)+\frac{4 V_{\text {dd }} I_{o} N_{r}}{f_{\text {APU }}}\left(C_{\text {sum }}+c_{\text {prod }}\right)$ \\
\hline$k_{3}$ & $T_{s} \frac{\xi}{\eta}$ & $T_{s} \frac{\xi}{\eta}$ \\
\hline$k_{4}$ & $\frac{A d^{\alpha} \sigma_{n}^{2} N_{f} N_{t}^{2}}{\epsilon_{\max }}$ & $\frac{A d^{\alpha} \sigma_{n}^{2} N_{r}}{\epsilon_{\max }}\left(N_{t}^{2}-\epsilon_{\max }\right)$ \\
\hline
\end{tabular}

where $k_{1}$ through $k_{4}$ are given in Table 2 . This optimization problem has a quadratic objective function with restrictions forming a convex domain. It is to be noted that (20a) is to be solved as an integer optimization problem, because $\frac{N_{p}}{N} \in \mathbb{N}$ must be satisfied. We do this by first solving (20a) by means of Lagrange multipliers [11] in its continuous variable form (see Appendix) and then analyze the integer solution requirement.

The optimal values of the number of pilot symbols $N_{p}^{*}$ and transmission power $P_{\mathrm{tx}}^{*}$ depend on constant $k_{4}$ :

- If $N P_{\max } \geq k_{4}$, then the constraints (20b) and (20c) are active. Therefore,

$$
\begin{aligned}
& N_{p}^{*}=N \\
& P_{\mathrm{tx}}^{*}=\frac{k_{4}}{N}
\end{aligned}
$$

- If $N P_{\max } \leq k_{4}$, then the constraints (20b) and (20d) are active for the non-integer optimization problem, so that $P_{\mathrm{tx}}=P_{\max }$ and $N_{p}=\frac{k_{4}}{P_{\max }}$. By imposing the integer constraint over $N_{p}$, we find

$$
\begin{aligned}
& N_{p}^{*}=N\left\lceil\frac{k_{4}}{N P_{\max }}\right\rceil \\
& P_{\mathrm{tx}}^{*}=\frac{k_{4}}{N_{p}^{*}},
\end{aligned}
$$

where $\lceil x\rceil$ denotes the smallest integer larger than $x$.

The MSE constraint (20b) is active in both cases above because it locks the trade-off between the optimal transmission power $P_{\mathrm{tx}}^{*}$ and the optimal number of pilot symbols $N_{p}^{*}$.

The condition $k_{4}=N P_{\max }$ separates the optimal solution into the two regions described above. As shown in Table $2, k_{4}$ depends on several parameters and the dependence of the optimality of $N_{p}$ and $P_{\mathrm{tx}}$ on them may be analyzed for each one. For illustration, we provide the analysis for how $N_{p}^{*}$ and $P_{\mathrm{tx}}^{*}$ vary with distance. Considering $d$ as a variable, condition $k_{4}=N P_{\max }$ defines a critical distance $d_{c}$. For $d \leq d_{c}$ the optimal solution is to transmit the minimum number of pilot symbols $\left(N_{p}^{*}=N\right)$, and for $d>d_{c}$, the solution is to use $P_{\mathrm{tx}} \approx P_{\max }$ (this relationship is approximated because of the ceiling function in (22a)). For the LS algorithm, the critical distance is

$$
d_{\mathrm{c}, \mathrm{LS}}=\left(\frac{P_{\max } \epsilon_{\max }}{A \sigma_{n}^{2} N^{2}}\right)^{\frac{1}{\alpha}}
$$

and for the MMSE algorithm it is

$$
d_{\mathrm{c}, \mathrm{MMSE}}=\left(\frac{P_{\max } \epsilon_{\max }}{A \sigma_{n}^{2}\left(N^{2}-\epsilon_{\max }\right)}\right)^{\frac{1}{\alpha}} .
$$

\section{Numerical evaluation}

In order to provide numerical examples that allow for comparing the minimum energy consumption of each estimator, we use the parameters given in [5] for a generic low-power transceiver (summarized in Table 3) and the parameters given in $[12,13]$ for a standard APU (Table 4). Finally, we choose $N=4$ antennas for both transceivers.

Figures 3 and 4 show the optimal number of pilot symbols and transmitted power for a maximum estimation MSE of $-10 \mathrm{~dB}$ for each algorithm. For this estimation error, the critical distance for the LS and the MMSE algorithms is about $42 \mathrm{~m}$ (dashed line). In both cases, the number of pilot symbols remains constant (Figure 3 ) while

Table 3 Generic low-power device parameters

\begin{tabular}{lll}
\hline Parameter & Description & Value \\
\hline$W$ & Bandwidth & $10 \mathrm{kHz}$ \\
$T_{s}$ & Symbol period & $100 \mu \mathrm{s}$ \\
$P_{\text {syn }}$ & Frequency synthesizer power & $50 \mathrm{~mW}$ \\
& consumption & \\
$T_{\mathrm{tr}}$ & Frequency synthesizer settling & $5 \mu \mathrm{s}$ \\
$\check{P}_{\mathrm{el}, \mathrm{tx}}$ & time & \\
$\check{P}_{\mathrm{el}, \mathrm{rx}}$ & Tx electric power consumption & $98.2 \mathrm{~mW}$ \\
$\alpha$ & Rx electric power consumption & $112.5 \mathrm{~mW}$ \\
$A$ & Path loss coefficient & 3.5 \\
$N_{0}$ & Channel path loss & $30 \mathrm{~dB}$ \\
$N_{\mathrm{f}}$ & Noise power density & $-174 \mathrm{dBm} / \mathrm{Hz}$ \\
$M_{\mathrm{l}}$ & Receiver noise figure & $10 \mathrm{~dB}$ \\
$\eta$ & Link margin & $40 \mathrm{~dB}$ \\
\hline & PA drain efficiency & 0.35 \\
\hline
\end{tabular}


Table 4 Technical parameters of an arithmetic and logic unit (ALU)

\begin{tabular}{lll}
\hline Parameter & Description & Value \\
\hline$f_{\mathrm{ALU}}$ & ALU frequency & $20 \mathrm{MHz}$ \\
$V_{\mathrm{dd}}$ & ALU voltage & $3 \mathrm{~V}$ \\
$l_{0}\left(V_{\mathrm{dd}}, f_{\mathrm{ALU}}\right)$ & Average current & $6.37 \mathrm{~mA}$ \\
$c_{\text {sum }}$ & Adding cost & 6 cycles \\
$c_{\text {prod }}$ & Product cost & 13 cycles \\
$c_{\text {div }}$ & Division cost & 21 cycles \\
\hline
\end{tabular}

the transmitted power increases (Figure 4). At the critical distance, $N_{p}^{*}$ steps up to the next integer multiple of $N$, as described by (22a). At that point, the optimal transmission power steps back as shown by the sawtooth curves in Figure 4. As the distance increases further, the optimal transmitted power tends to $P_{\max }$.

In order to compare the energy required by both algorithms, we evaluate (14) and (17) at $N_{p}^{*}$ and $P_{\mathrm{tx}}^{*}$ (Figure 5). The energy consumption of both algorithms is identical for any practical purpose. However, the LS algorithm is simpler to implement because it does not require knowledge of the channel statistics and of the power of the noise. Therefore, it is the preferred choice for attaining a given estimation quality (MSE) at minimum energy consumption.

For a standard low-power device, the maximum operation distance is about $50 \mathrm{~m}$ [14]. Figure 5 shows that at this distance each antenna must send two pilot symbols in order to estimate the channel with an MSE of $-10 \mathrm{~dB}$ with minimum energy consumption. On the other hand, given that each antenna transmits two preamble symbols, the maximum link distance (which is achieved maximizing the transmission power) is about $53 \mathrm{~m}$.

If we consider a minimum detectable signal-to-noise ratio (SNR) of $7 \mathrm{~dB}$, the maximum link distance allowed by the generic system used here as example is about $123 \mathrm{~m}$ (Figure 6). At this distance, the channel estimators require 128 pilot symbols for an estimation MSE of $-10 \mathrm{~dB}$, and they consume 10 times more energy than in the 53-m link case. This suggests that under certain conditions, it may be more efficient in terms of energy consumption to use multi-hop MIMO communications. However, that analysis must incorporate the energy consumption required for transmitting correctly a message [2] and lies beyond the scope of this study.

Figure 7 shows the minimum energy consumption of each algorithm as a function of the estimation MSE. As expected, the minimum energy drops in steps as the error tolerance grows. It is to be noted that near each energy step, slight estimation performance degradationand hence slight error rate degradation-can be traded for significant reduction in energy consumption for channel estimation.

\section{Conclusions}

In this study, we present a methodology for determining the length and transmission power of training signals that allow for producing estimates of MIMO channels with a given estimation error and minimal joint energy

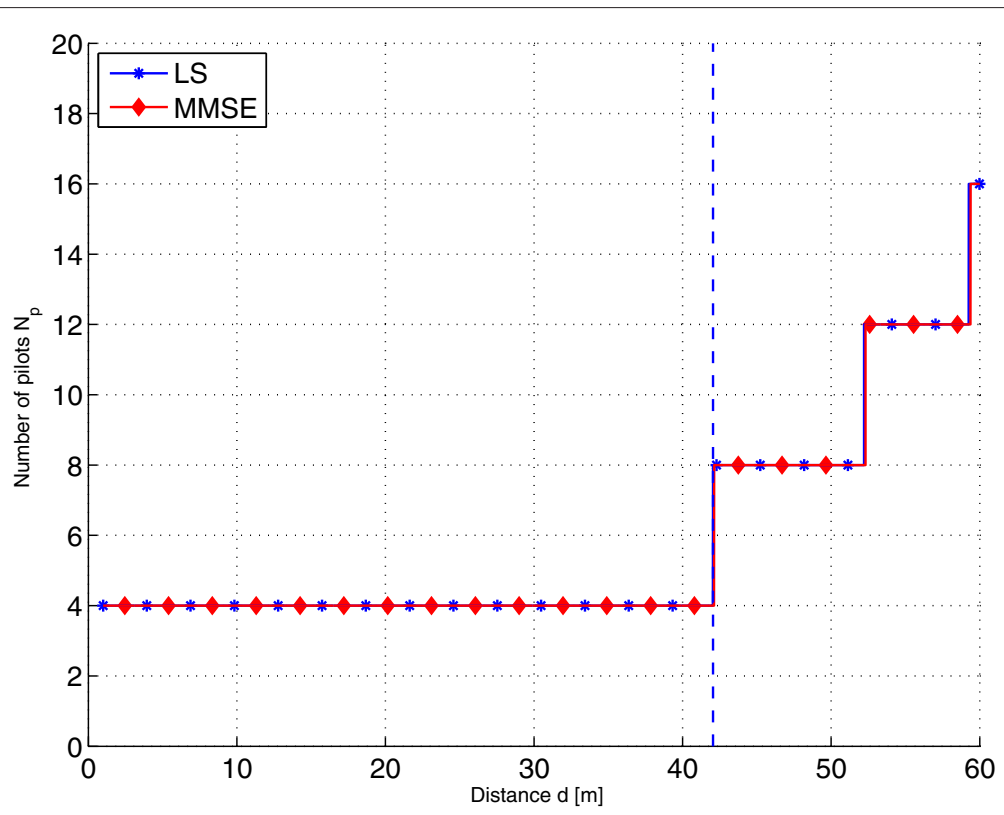

Figure 3 Optimal number of pilot symbols versus distance for the LS and MMSE algorithms with estimation MSE of $-10 \mathrm{~dB}$. 


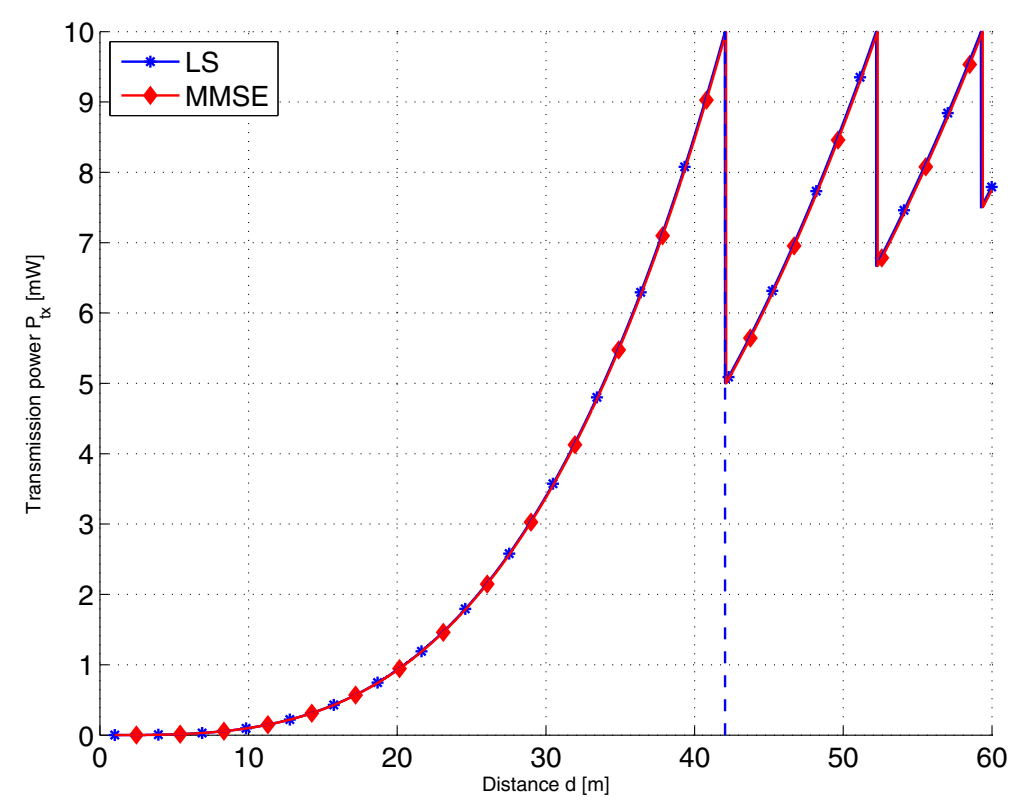

Figure 4 Optimal transmission power versus distance for the LS and MMSE algorithms with estimation MSE of $-10 \mathrm{~dB}$.

consumption among transmitter and receiver. We develop a general energy consumption model for the complete process of channel estimation. The model includes energy consumption due to transmission and reception of the training signals and due to the processing required to obtain the channel estimates.

The model was used for studying and optimizing the energy consumption of the LS and MMSE channel estimation algorithms. Both algorithms consume virtually the same energy when operated at their respective optimal training signal configurations of length and transmission power. However, the LS algorithm does not require the knowledge of the channel statistics and of the power of the noise, which makes the LS algorithm the preferred choice.

For link distances of about $50 \mathrm{~m}$, our results show that the channel estimation with minimum energy is achieved using two preamble symbols per transmit antenna when

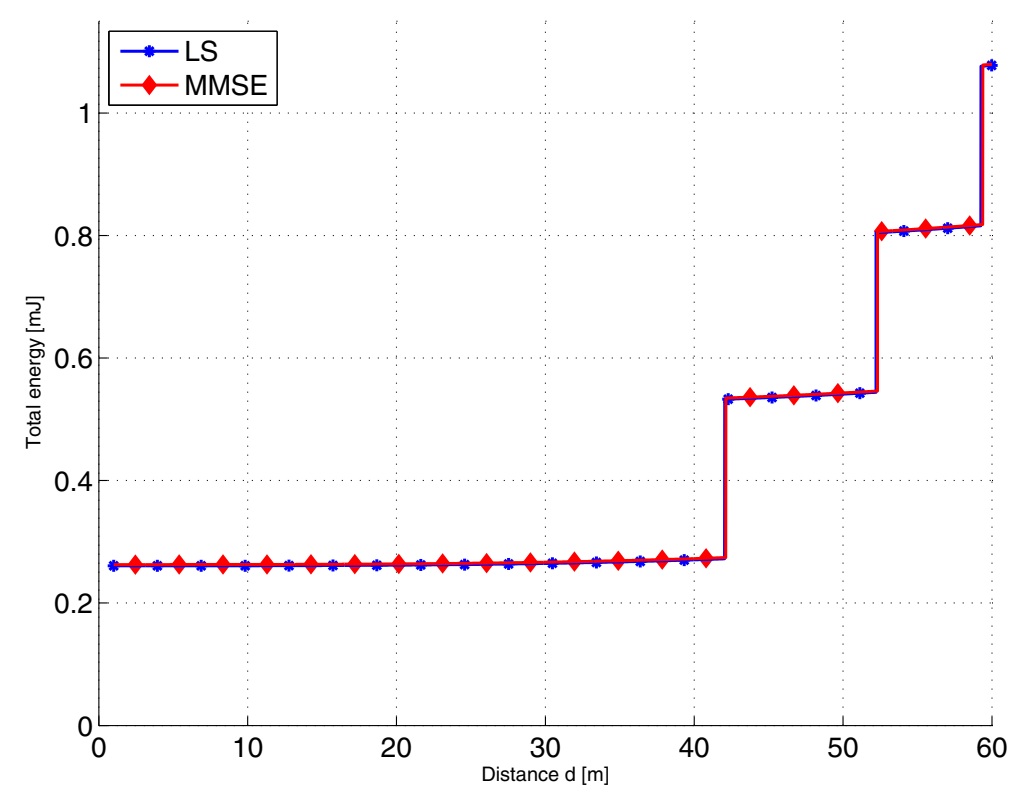

Figure 5 Minimum energy consumed by the LS and MMSE algorithms, with estimation MSE of $-10 \mathrm{~dB}$. 


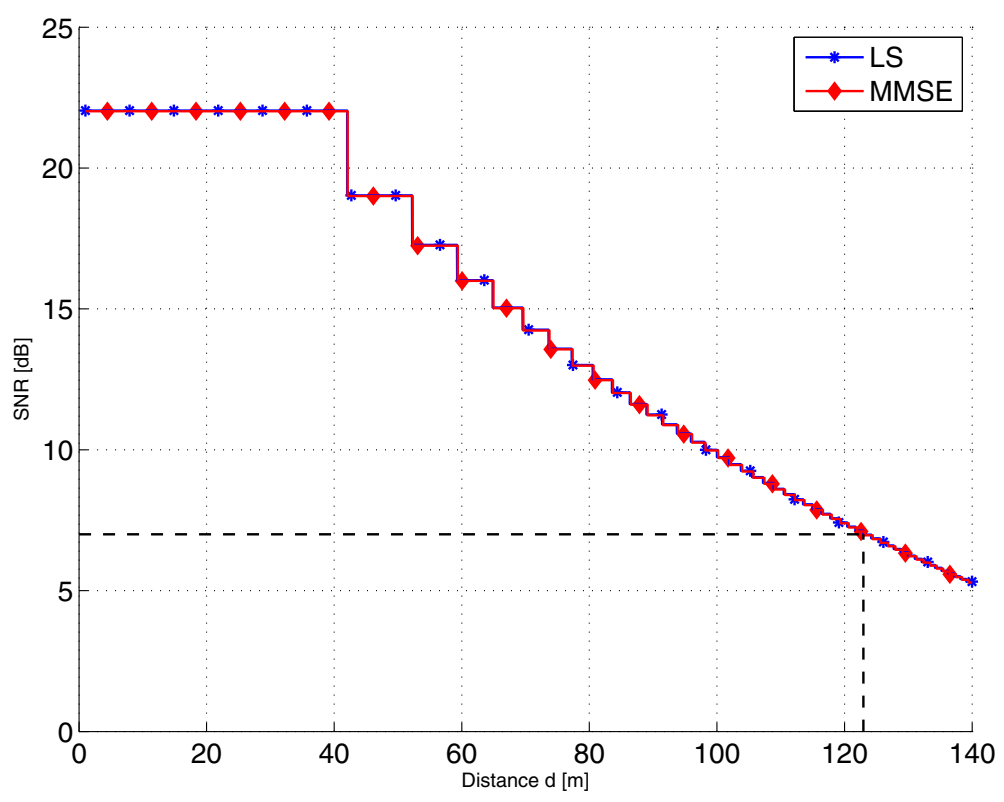

Figure 6 SNR at the input of the channel estimator. The vertical dashed line shows the maximum distance for an SNR of $7 \mathrm{~dB}$.

the target estimation MSE is $-10 \mathrm{~dB}$. For distances of approximately $120 \mathrm{~m}$, the minimum energy consumption required to achieve the same estimation quality increases tenfold for both algorithms due to path loss. This indicates that longer range MIMO communications can be performed more energy-efficiently by multi-hop routes than over single-hop links.

\section{Appendix}

\subsection{Optimization problem}

We define an auxiliary variable $N_{u}=\frac{N_{p}}{N} \in \mathbb{N}$. The Lagrangian of the optimization problem (20a) is

$$
\begin{aligned}
\mathcal{L}= & k_{1}+k_{2} N N_{u}+k_{3} N N_{u} P_{\mathrm{tx}}+\lambda_{1}\left(-N N_{u} P_{\mathrm{tx}}+k_{4}\right) \\
& +\lambda_{2}\left(-N_{u}+1\right)+\lambda_{3}\left(P_{\mathrm{tx}}-P_{\max }\right)
\end{aligned}
$$

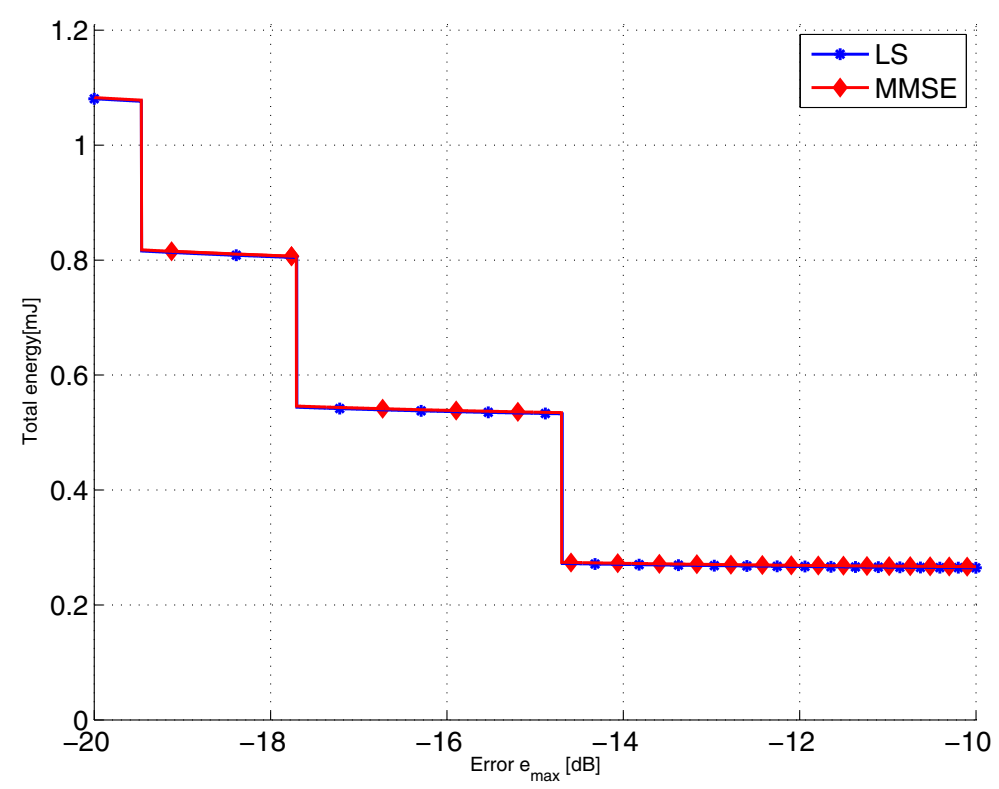

Figure 7 Minimum energy consumed by the LS and MMSE algorithms versus mean square estimation error for a link distance of $30 \mathrm{~m}$. 
with multipliers $\lambda_{1}, \lambda_{2}$, and $\lambda_{3}$. After taking derivative of $\mathcal{L}$ with respect to $\lambda_{1}, \lambda_{2}, \lambda_{3}, N_{u}$, and $P_{\mathrm{tx}}$ we find two feasible solutions:

- Case I, $\lambda_{1} \neq 0, \lambda_{2} \neq 0, \lambda_{3}=0$

The R1 (20b) and R2 (20c) constraints are active, therefore

$$
\begin{aligned}
& N_{u}^{*}=1 \\
& P_{\mathrm{tx}}^{*}=\frac{k_{4}}{N} .
\end{aligned}
$$

This occurs when $k_{4} \leq N P_{\max }$. In this case, $N_{p}^{*}=N$ is a feasible integer solution.

- Case II, $\lambda_{1} \neq 0, \lambda_{2}=0, \lambda_{3} \neq 0$

The R1 (20b) and R3 (20d) constraints are active, therefore, $P_{\mathrm{tx}}=P_{\max }$ and $N_{u}=\frac{k_{4}}{N P_{\max }}$. This occurs when $k_{4} \geq N P_{\max }$, but now $N_{u}$ is not necessarily a natural number. Incorporating this constraint, we obtain $N_{u}^{*}=\left\lceil\frac{k_{4}}{N P_{\max }}\right\rceil$. Therefore,

$$
\begin{aligned}
& N_{p}^{*}=N\left\lceil\frac{k_{4}}{N P_{\max }}\right\rceil \\
& P_{\mathrm{tx}}^{*}=\frac{k_{4}}{N_{p}^{*}} .
\end{aligned}
$$

\section{Competing interests}

The authors declare that they have no competing interests.

\section{Acknowledgements}

This study was funded by a Master's degree scholarship and by projects FONDECYT 1110370 and FONDEF D0911094 from CONICYT.

Received: 21 June 2012 Accepted: 23 October 2012

Published: 26 November 2012

\section{References}

1. S Cui, A Goldsmith, A Bahai, Energy-efficiency of MIMO and cooperative MIMO techniques in sensor networks. IEEE J. Sel. Areas Commun. 22(6), 1089-1098 (2004)

2. F Rosas, C Oberli, in PIMRC2012-Track 1: PHY and Fundamentals (PIMRC2012-PHY) Vol. 1. Energy-efficient MIMO SVD communications, (Sydney, Australia, 2012), pp. 1582-1587

3. M Biguesh, A Gershman, Training-based MIMO channel estimation: a study of estimator tradeoffs and optimal training signals. IEEE Trans. Signal Process. 54(3), 884-893 (2006)

4. B Hassibi, B Hochwald, How much training is needed in multiple-antenna wireless links. IEEE Trans. Inf. Theory. 49(4), 951-963 (2003)

5. S Cui, A Goldsmith, A Bahai, Energy-constrained modulation optimization. IEEE Trans. Wirel. Commun. 4(5), 2349-2360 (2005)

6. A Sinha, AP Chandrakasan, in IEEE Design Automation Conference, Vol. 617 JouleTrack: a web based tool for software energy profiling, (Las Vegas, United States, 2001), pp. 220-225

7. S Yatawatta, A Petropulu, C Graff, in IEEE International Conference on Acoustics, Speech, and Signal Processing, 2005 (ICASSP'05) Vol. 4. Energy efficient channel estimation in MIMO systems, (Philadelphia, United States, 2005), pp. 317-320

8. TRappaport, Wireless Communications: Principles and Practice. (Prentice Hall Communications Engineering and Emerging Technologies Series, Prentice Hall PTR, 2002)

9. A Sayed, Adaptive Filters. (Wiley \& Sons, New York, 2011)
10. J Conway, Functions of One Complex Variable, Volume 1 of Graduate Texts in Mathematics. (Springer Verlag, Berlin, 1978)

11. CMuñoz, Señales de entrenamiento óptimas para estimación de canal MIMO energéticamente eficiente. Master's thesis, Pontificia Universidad Católica de Chile, 2012

12. T Instrument, MSP430x2xx Family. User's guide (2012), http://www.ti. com/lit/ug/slau144i/slau144i.pdf

13. K Venkat, Efficient multiplication and division using MSP430. Application report (2006), http://www.ti.com/lit/an/slaa329/slaa329.pdf

14. D Gislason, ZigBee Wireless Networking. (Elsevier, Amsterdam, 2008)

\section{doi:10.1186/1687-1499-2012-353}

Cite this article as: Muñoz and Oberli: Energy-efficient estimation of a MIMO channel. EURASIP Journal on Wireless Communications and Networking 2012 2012:353.

\section{Submit your manuscript to a SpringerOpen ${ }^{\mathcal{O}}$ journal and benefit from:}

- Convenient online submission

- Rigorous peer review

- Immediate publication on acceptance

- Open access: articles freely available online

- High visibility within the field

- Retaining the copyright to your article

Submit your next manuscript at $>$ springeropen.com 